\title{
The IoT and registration of MRI brain diagnosis based on genetic algorithm and convolutional neural network
}

\author{
Ahmed Shihab Ahmed ${ }^{1}$, Hussein Ali Salah \\ ${ }^{1}$ Department of Basic Sciences, College of Nursing, University of Baghdad, Baghdad, Iraq \\ ${ }^{2}$ Department of Computer Systems, Technical Institute-Suwaira, Middle Technical University, Baghdad, Iraq
}

\begin{tabular}{l} 
Article Info \\
Article history: \\
Received Jul 16, 2021 \\
Revised Oct 24, 2021 \\
Accepted Nov 26, 2021 \\
\hline
\end{tabular}

Keywords:

Arduino global system for mobile

Convolution neural network

Discrete wavelet transform

Genetic algorithm

Internet of things

Registration of magnetic

resonance imaging brain

\begin{abstract}
The technology of the multimodal brain image registration is the key method for accurate and rapid diagnosis and treatment of brain diseases. For achieving high-resolution image registration, a fast sub pixel registration algorithm is used based on single-step discrete wavelet transform (DWT) combined with phase convolution neural network $(\mathrm{CNN})$ to classify the registration of brain tumors. In this work apply the genetic algorithm and CNN clasifcation in registration of magnetic resonance imaging (MRI) image. This approach follows eight steps, reading the source of MRI brain image and loading the reference image, enhencment all MRI images by bilateral filter, transforming DWT image by applying the DWT2, evaluating (fitness function) each MRI image by using entropy, applying the genetic algorithm, by selecting the two images based on rollout wheel and crossover of the two images, the CNN classify the result of subtraction to normal or abnormal, "in the eighth one," the Arduino and global system for mobile (GSM) 8080 are applied to send the message to patient. The proposed model is tested on MRI Medical City Hospital in Baghdad database consist 550 normal and 350 abnormal and split to $80 \%$ training and 20 testing, the proposed model result achieves the $98.8 \%$ accuracy.
\end{abstract}

This is an open access article under the CC BY-SA license.

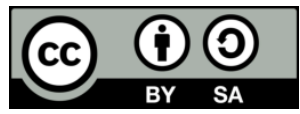

\section{Corresponding Author:}

Hussein Ali Salah

Department of Computer Systems, Technical Institute-Suwaira, Middle Technical University

Muasker Al Rashid Street, Baghdad, Iraq

Email: hussein_tech@mtu.edu.iq

\section{INTRODUCTION}

Currently, medical imaging systems have a crucial role in the clinical workflow, due to their ability to reflect anatomical and physiological features which are not otherwise available for inspection [1], [2]. Medical image technology uses a variety of different concepts to quantify the spatial distributions of the physical characteristics of humans and help to better understanding to complex or unusual diseases. Data processing is essential for computer assistance medical diagnose [3], [4]. The method to integrate complementary information from more than one image of a certain organ into one composite image can provide useful information. The number of available modalities and the data volume of data in medical images makes it very difficult to explicitly use them at different levels of complementary data [5], [6]. Moreover, each method offers a partial amount of knowledge, and often two or more modes from the same patient are employed to get well-understood sensed material. The first one can provide decent structural details (i.e. brilliant contrast to the bones) is computed tomography (CT) scanner, while the magnetic resonance imaging (MRI) provides good data on weak tissue (soft tissue). Two modalities are frequently used in brain visualization (such as white matter and grey matter [7]-[9]. The word "registration" illustrates that is, finding a match between two image registration is used to determine geometric transitions to provide a normal or reference image in the created 
image [10], [11]. The technique of registration of images can be divided into three types, the optimization of similarity measures, geometric transformation, and interpolation. The measure of similarity represents the key step in the recording of images [12]-[14]. The registration procedure is of immense importance. MRI is currently the most important way of obtaining soft tissue imaging especially in oncology, since the image contrasts and resolution of lesions and healthy tissue are significantly improved [15], [16]. The MRI is considered to be more accurate to assess the level of cancer infiltration than computed tomography [17]-[19]. The registration of biomedical images has many approaches, gold standard uses region-of-interest markers, and other methods include correlation of geometrical characteristics [20], [21]. Intensity-based methods are more worked in recent years to quantify correlations between an image with the intensity values (color or gray level). The consistency of recording medical images depends on the options made using the method of processing, interpolation, similarity calculation, and optimization. A specific use of the genetic algorithm is the primary original characteristic of the method (from encoding to genetic space screening) [22], [23]. Genetic algorithm (GA) relies upon "survival of the fittest" principle and a global selection of the best for the new generation by crossover and mutation operators select the world's best new generation. The optimization scheme is initialized by updating the generations with a random population of solutions and searches for optima [24], [25]. Neural networks are playing a significant part in medical diagnosis and classification of brain and tumors diseases. The neural network methods were implemented to relay the neural architecture of the image segmentation network, also a hybrid image segmentation neural network with fuzzy [26], [27].

The main motivations of this work is incremental growth in the internet of things (IoT) technology to be anywhere, anytime results in increasing demand for automation in e-health. The need for automatic diagnosis applications with less time complexity and accuracy is highly preferred. Big data and data science are a new hot topic addressed by soft computing techniques for their applicability to deal with vagueness and uncertain data besides learning capability. The objectives of this work to develop a transmission model for the IoT environment based on the cellular network that enables clinical diagnostic automation. The main contributions is developing a MRI algorithm based on wavelet and fusion technology inside GA with convolution neural network (CNN) for detection high accuracy of the proposed work. The main problem of work is introduce automatic system for detection and daignosis MRI brain with high accuracy. In this study, a hybrid system was proposed, which consists of two stages, the first stage is image registration that includes the genetic algorithm, and the second stage is image detection that includes CNN and connected in by using global system for mobile (GSM8080) for send massage to patient an IoT environment. This work aims to develop a soft computing model for image registration as a first stage in the automatic diagnosis system. Then, it proposes and incorporates a detection stage to automate the diagnosis process, which will prove the accuracy of the proposed registration stage in the clinical workflow based on the IoT environment.

\section{RELATED WORKS}

Anaraki et al. [28], proposed a CNN-based method and genetic algorithm for classifying various grading of glioma by MRI. In the proposed method, CNN's architecture is developed by the use of a genetic algorithm, as opposed to current techniques of selection the (DNN) architecture, which relies upon on trial and error or through the adoption common structures that are defined in advance. Furthermore, to minimize prediction error variance, bagging as an ensemble algorithm was used on the optimum model that genetic algorithm developed. To indicate the results briefly, in one case study, a $90.9 \%$ accuracy is gotten to classify three grades of glioma in different case study, Pituitary, Meningioma, and Glioma tumor types are categorized with the total accuracy at 94.2\%. Shahamat and Abadeh [29], introduced 3D-CNN for classifying brain magnetic resonance imaging into two pre-determined classifications. Moreover, a method of genetic algorithm based brain masking was suggested as a visualization technique providing a clear understanding to three-dimension convolutional neural network function. This method is composed two steps. In the first one, a set of brain MRI scans will be utilized for training the three-dimension convolutional neural network. In the second one, a genetic algorithm is implemented to detect brain regions in MRI scanning. The regions are brain areas mostly used by 3D-CNN for extracting significant and discriminative traits from these areas. To apply GA to magnetic resonance imaging scans of brain, a new approach of chromosomal encoding is suggested. Furthermore, an evaluation is conducted to this proposed framework by the use Alzheimer's disease Neuroimaging initiative (ADNI) (including one hundred forty individuals to disease classification of Alzheimer) and autism brain imaging data exchange (ABIDE) (including one thousand individuals for Autism classification) brain MRI datasets. Experimental results showed a five-fold classification accuracy of 0.70 for the dataset of Autism brain imaging data exchange and 0.85 for the dataset of Alzheimer's disease Neuroimaging initiative. Those regions are interpreted as brain segments, which 3D-CNN typically uses to extract features to classify brain diseases. Experimental results showed that along with interpretability of 
model, this method increases the classification model's final performance in number of cases concerning the parameters of the model

Sajjad et al. [30] introduced multi-grade brain tumor classification system based CNN. Firstly: segmenting tumor regions from images of magnetic resonance imaging by the use of deep learning technique. Secondly: augmenting data widely can be used to train the system proposed in order to avoid any problem related to lacking data when handling with MRI to classify multi-graded brain tumors. Thirdly: a pre-trained CNN model is fine-tuned using augmented data for brain tumor grade classification. Thirdly, CNN model trained in advance is fine-tuned using by the use of augmented data to classify the degree of brain tumor.

Chang et al. [31] information related to MRI and molecular data, for 259 patients, from cancer imaging archives were obtained, those individuals were having glioma, either high or low-grade. CNN was trained for classifying $1 \mathrm{p} / 19 \mathrm{q}$ codeletion, isocitrate dehydrogenase 1 (IDH1) mutation status, and O6methylguanine-DNA methyltransferase (MGMT) promoter hypermethylation status. Principal component analysis of the final convolutional neural network layer was used to extract the key imaging features to classify cases accurately. Results: the process of classification is highly accurate: IDH1 mutation status, 94\%. The authors Rahman et al. [32] implemented IoT to facilitate farming, particularly for those who want a smart approach to agriculture. This study focuses on real-time surveillance with the low cost-effective security solution. Make the most of computer resources such encryption and decryption time, battery usage, and so on, divide the data utilized in the IoT environment into three categories of sensitivity: low, medium, and high sensitive data [33]. In this paper, a framework is provided for encrypting data based on the level of sensitivity utilizing machine learning K-nearest neighbors (K-NN). Tanh et al. [34] enhanced security protocols presented a viable solution for comprehensive protection of IoT systems from network security assaults. Algorithmic enhancement favorably contributes to this crucial work by combining security solutions on the levels of the IoT with code optimization. Also, enhance and combine the DTLS Protocol with the overhearing mechanism, and then conduct tests to demonstrate effectiveness, feasibility, cost-efficiency, and applicability on popular IoT network models. Presents NB-IoT testing approach that is tailored to the local radio network planning requirements [35]. Adducing the major findings about the viability of employing an in-band scenario for deploying NB-IoT over a $4 \mathrm{G}$ network in a suburban setting based on the acquired data. Rajbongshi et al. [36], Erwin et al. [37] suggested different types of leaf diseases, such as anthracnose, gall machi, powdery mildew, and red rust, are employed in the dataset, which includes 1500 photos of damaged and healthy mango leaves. A new category has been added to the dataset. Also looked at the overall performance matrices and discovered that the DenseNet201 beats other models by achieving the highest accuracy of $98.00 \%$. Fadil et al. [38] The medical images are enhanced using the fuzzy C-means clustering (FCM) approach. There are two stages to the enhancing procedure. On the picture pixels, the suggested technique performs a cluster test. The difference in gray level between the various items is then increased to achieve the medical picture enhancing goal. Various photos were used to test the experimental outcomes.

\section{THE PROPOSED MODEL COMPONENTS OF MRI BRAIN DIAGNOSIS}

In this proposed work, the genetic algorithm and CNN are used to determine the brain tumor classification based on the principle of registration and this is achieved by loading the (source and reference) image. After that, image is processed in regard with smoothing, reducing noise, by using Gaussian filter. Genetic algorithm is also applied to achieve the principle of registration, then, CNN is used to classify the brain tumor. Eventually, sending a massage to a patient explaining the tumor grade depending on GSM Arduino to achieve the principle of IoT, as shown in Figure 1 and Figure 2.

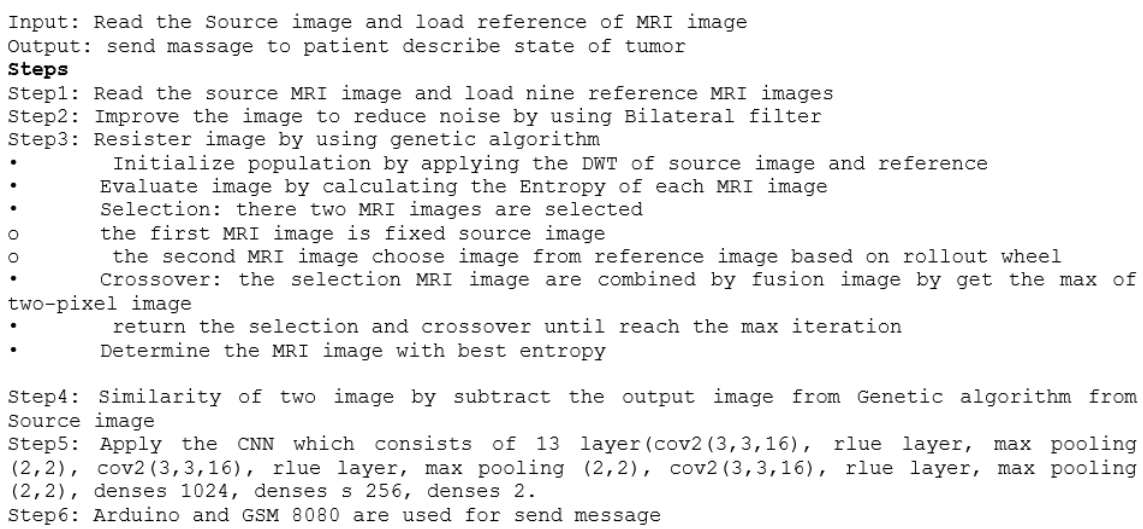

Figure 1. Describe the proposed approach of work 


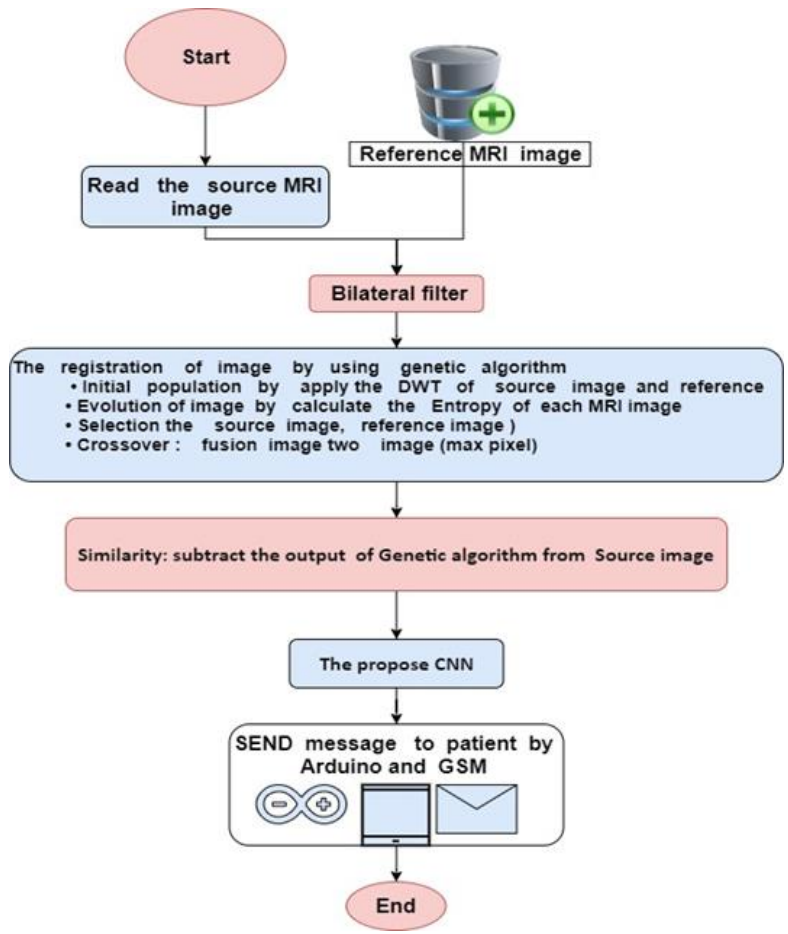

Figure 2. The work flow of MRI brain diagnosis

\section{MRI BRAIN DIAGNOSIS SYSTEM IMPLEMENTATION}

This work proposed the automatic model to detect the brain tumor and send a message to patient, that can achieved by using MATLAB 2020a and Arduino with GSM8080. It uses database from Medical City Hospital in Baghdad, for 80 patients, (800) images are diagnosed to two classes normal 55 persons and 35 patients. The source image and reference image are loaded as shown in Figure 3, the genetic algorithm is applied to achieve the registration, then the output of genetic algorithm is subtracted from source MRI image then, the database is divided to 80 training and 20 testing based on cross-validation. In addition to, the CNN is applied to classify the image and send it to patient by GSM as shown, in Figures 3-5.

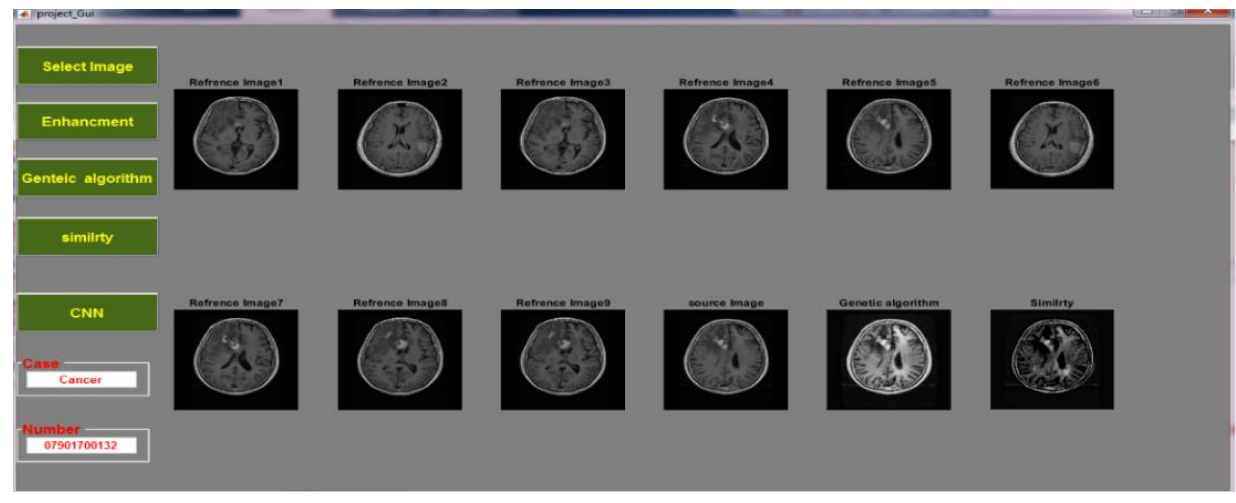

Figure 3. The Gui of Matlab show the result of proposed work

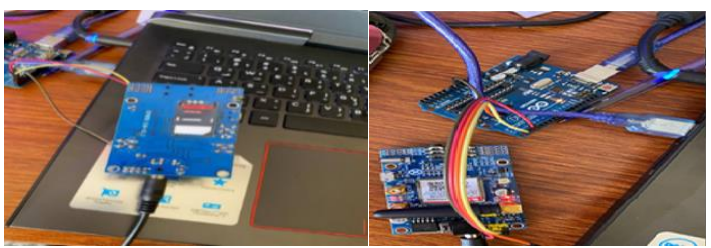

Figure 4. The Arduino and GSM are connected to Matlab 


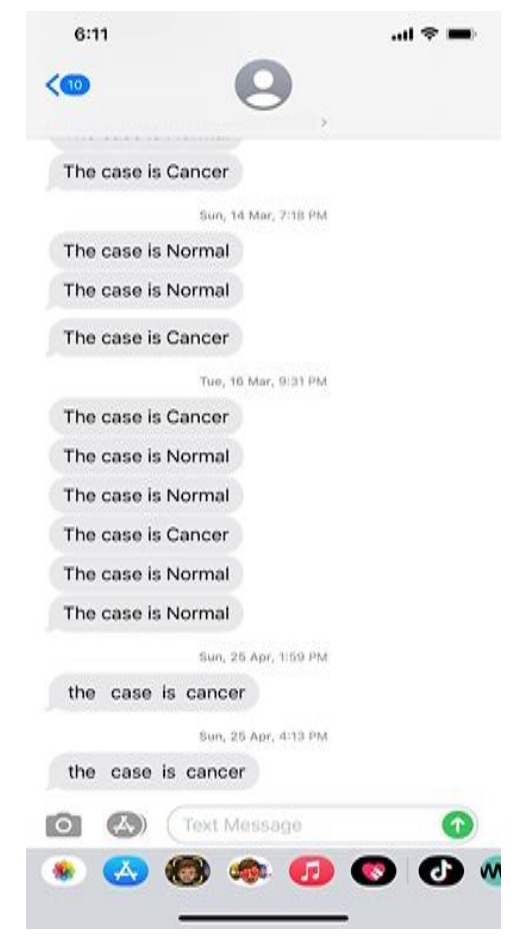

Figure 5. The message sent to patient

The CNN training model is introduced, the volume of entered MRI is $100 * 100 * 31$, and training setup of CNN works, as shown here, momentum is 0.9 and learning rate is 0.001 and the architecture of network is composed 4 pooling layers and 4 convolution layers and, two fully connected layers follow those layers. A Relu layer comes after a convolution layer, an activation function for improving the CNNs performance. In the network training, regularization with the weight decay five $\times$ ten-four was used. Initially, the learning rate was set to 0.001 , the training was stopped after 1000 epoch, and the dropout ratio was set to zero.0, as shown in Table 1.

Table 1. Analysis result of CNN model

\begin{tabular}{clcc}
\hline Layer & Name & Activations & Learnable \\
\hline 1 & Image input & $100 * 100 * 1$ & - \\
2 & Convolution1 & $96 * 96 * 1$ & Weight $5 * 5 * 1 * 20$, \\
3 & Relu1 & $96 * 96 * 1$ & Bias $1 * 1 * 20$ \\
4 & Pool max1 & $48 * 48 * 20$ & - \\
5 & Convolution2 & $44 * 44 * 20$ & - \\
6 & Relu2 & $44 * 44 * 20$ & Beight $5 * 5 * 20 * 20$ \\
7 & Pool max2 & $22 * 22 * 20$ & - \\
8 & Fully Connected Layer & $1 * 1 * 1024$ & - \\
9 & Fully Connected Layer & $1 * 1 * 256$ & - \\
10 & Fully Connected Layer & $1 * 1 * 2$ & - \\
11 & SoftMax Layer & $1 * 1 * 2$ & - \\
12 & Classification Layer & - & - \\
\hline
\end{tabular}

After building the network architecture as shown in Table 2, the hybrid Mamdani fuzzy and CNN train model starts in epoch (1), the parameter of Elapsed time is 2 second, parameter of accuracy is $28.13 \%$ and the parameter of mini batch loss is 1.4149 . At the epoch 14 the parameter of accuracy reached to $92.19 \%$, parameter of mini batch loss 0.2024 and the elapsed time is 05:41 minute. At the epoch 28 the parameter of accuracy reached to $99.22 \%$, parameter of mini batch loss 0.0576 and the elapsed time is 11:08 minute. At the epoch 41 the parameter of accuracy reached to $100 \%$, parameter of mini batch loss 0.0021 and the elapsed time is 16.32 minute.

After training the models for recognition of a brain tumor, the classification results are as shown in Table 3, a detailed classification of the test samples is listed. The true and reference columns represent the 
true situation, while the row values are the predicted true, the model or the model has to predict false as shown in Table 3. In Table 4 present the compare the proposed work with other researcher.

Table 2. Show the train on CNN

\begin{tabular}{cccccc}
\hline Epoch & Iteration & $\begin{array}{c}\text { Time Elapsed } \\
\text { (hh:mm: ss) }\end{array}$ & $\begin{array}{c}\text { Mini-batch } \\
\text { Accuracy }\end{array}$ & $\begin{array}{c}\text { Mini-batch } \\
\text { loss }\end{array}$ & $\begin{array}{c}\text { Base Learning } \\
\text { Rate }\end{array}$ \\
\hline 1 & 1 & $00: 00: 02$ & $28.13 \%$ & 1.4149 & 0.0010 \\
5 & 50 & $00: 01: 52$ & $46.06 \%$ & 0.9088 & 0.0010 \\
10 & 100 & $00: 03: 43$ & $84.38 \%$ & 0.4804 & 0.0010 \\
14 & 150 & $00: 05: 41$ & $92.19 \%$ & 0.2024 & 0.0010 \\
19 & 200 & $00: 07: 32$ & $98.44 \%$ & 0.1102 & 0.0010 \\
23 & 250 & $00: 09: 20$ & $86.72 \%$ & 0.3928 & 0.0010 \\
28 & 300 & $00: 11: 08$ & $99.22 \%$ & 0.0576 & 0.0010 \\
32 & 350 & $00: 12: 56$ & $100.00 \%$ & 0.0936 & 0.0010 \\
37 & 400 & $00: 14: 44$ & $100.00 \%$ & 0.0361 & 0.0010 \\
41 & 450 & $00: 16: 32$ & $100.00 \%$ & 0.0021 & 0.0010 \\
46 & 500 & $00: 18: 20$ & $100.00 \%$ & 0.0003 & 0.0010 \\
50 & 550 & $00: 20: 03$ & $100.00 \%$ & 0.0005 & 0.0010 \\
55 & 600 & $00: 21: 46$ & $100.00 \%$ & 0.0003 & 0.0010 \\
60 & 650 & $00: 23: 41$ & $100.00 \%$ & 0.0003 & 0.0010 \\
64 & 700 & $00: 25: 39$ & $100.00 \%$ & 0.0002 & 0.0010 \\
69 & 750 & $00: 27: 31$ & $100.00 \%$ & 0.0002 & 0.0010 \\
73 & 800 & $00: 29: 24$ & $100.00 \%$ & 0.0003 & 0.0010 \\
78 & 850 & $00: 31: 18$ & $100.00 \%$ & 0.0002 & 0.0010 \\
82 & 900 & $00: 33: 12$ & $100.00 \%$ & 0.0002 & 0.0010 \\
\hline
\end{tabular}

Noted

Training on single CPU

Initialization image normalization

Table 3. Test phase statistic measures for the CNN

\begin{tabular}{clc}
\hline Statistic & Description & CNN \\
\hline Accuracy & Rate of correctly predicted & $98.88 \%$ \\
& ACC= TP+ TN/(TP+ TN+ FP+ FN) & 55 \\
True positive & Number of correctly predicted. & 34 \\
True Negative & Number of malicious object which are correctly classified & 0 \\
False positive & Number of incorrectly predicted & 4 \\
False Negative & Number of malicious object which are incorrectly predicted & 1.12 \\
Misclassification Rate & the percentage of incorrectly predicted & Misclassification Rate =(FP+FN)/total \\
Specificity & calculated as the number of correct negative predictions Specificity= TN/(TN+FP) & 0.9814 \\
Precision & calculated as the number of correct positive & 1 \\
& Precision =TP /(TP+FP) & \\
\hline
\end{tabular}

Table 4. Compare the proposed work with other work

\begin{tabular}{ccl}
\hline Author & Accuracy & Methods \\
\hline Anaraki et al. [28] & $94.2 \%$ & GA-CNN \\
Zacharaki et al. [39] & $85 \%$ & Svm+Knn \\
Cheng et al. [40] & $91.28 \%$ & Svm+Knn \\
Paul et al. [41] & $91.43 \%$ & CNN \\
Afshar et al. [42] & $90.89 \%$ & CNN \\
Ertosun and Rubin [43] & $96 \%$ & CNN \\
Sultan et al. [44] & 96.13 & CNN \\
Chandra and Bajpai [45] & - & fractional filter (mask design) for benign brain tumor detection \\
Swati et al. [46] & $94.82 \%$ & pre-trained deep CNN model and propose a block-wise fine-tuning strategy \\
& & based on transfer learning \\
Proposed work & $98.8 \%$ & Genetic Algorithm and Convolution Neural Network \\
\hline
\end{tabular}

\section{CONCLUSION}

This work proposes building automatic IoT to detect and classify brain MRI based on deep learning and arduino GSM. Moreover, the principle of registration is applied to MRI using genetic algorithm, as following, reading the source image and loading the reference mage, reducing the noise of MRI image by bilateral filter, the genetic algorithm is applied to obtain the best fusion image from source and reference image, computing the similarly by subtracting the result of registration image to get the best feature of image, CNN is applied to classify brain tumor, and sending message to patient by GSM. The proposed model is 
tested on MRI Medical City Hospital in Baghdad, database consists of 550 normal and 350 abnormal images and split to $80 \%$ training and 20 testing, the proposed model result achieves the $98.8 \%$ accuracy. In the future work we can apply the IoT technique and registration of skin cancer based on K-means cluster and self organizing maps by using a data set of medical images.

\section{REFERENCES}

[1] H. Zaidi and I. El Naqa, "PET-guided delineation of radiation therapy treatment volumes: a survey of image segmentation techniques," European journal of nuclear medicine and molecular imaging, vol. 37, no. 11, pp. 2165-2187, 2010, doi: 10.1007/s00259-010-1423-3.

[2] N. Prasath, V. Pandi, S. Manickavasagam, and P. Ramadoss, "A comparative and comprehensive study of prediction of Parkinson's disease," Indonesian Journal of Electrical Engineering and Computer Science, vol. 23, no. 3, pp. 1748-1760, September 2021, doi: 10.11591/ijeecs.v23.i3.pp1748-1760.

[3] S. Mitra and B. U. Shankar, "Medical image analysis for cancer management in natural computing framework," Information Sciences, vol. 306, pp. 111-131, Sept. 2015, doi: 10.1016/j.ins.2015.02.015.

[4] A. A. Abbood, Q. M. Shallal, and M. A. Fadhel, "Automated brain tumor classification using various deep learning models: A comparative study," Indonesian Journal of Electrical Engineering and Computer Science, vol. 22, no. 1, pp. 252-259, Apr. 2021, doi: 10.11591/ijeecs.v22.i1.pp252-259.

[5] R. Singh and A. Khare, "Fusion of multimodal medical images using Daubechies complex wavelet transform-A multiresolution approach," Information fusion, vol. 19, pp. 49-60, 2014, doi: 10.1016/j.inffus.2012.09.005.

[6] N. H. I. M. Zaihani, R. Roslan, Z. Ibrahim, and K. A. F. A. Samah, "Automated segmentation and detection of T1-weighted magnetic resonance imaging brain images of glioma brain tumor," Bulletin of Electrical Engineering and Informatics, vol. 9, no. 3, pp. 1032-1037, June 2020, doi: 10.11591/eei.v9i3.2079.

[7] V. Laurent, G. Trausch, O. Bruot, P. Olivier, J. Felblinger, and D. Régent, "Comparative study of two whole-body imaging techniques in the case of melanoma metastases: advantages of multi-contrast MRI examination including a diffusion-weighted sequence in comparison with PET-CT," European journal of radiology, vol. 75, no. 3, pp. 376-383, 2010, doi: 10.1016/j.ejrad.2009.04.059.

[8] J. L. Elman, "On the meaning of words and dinosaur bones: Lexical knowledge without a lexicon," Cognitive science, vol. 33, no. 4, pp. 547-582, 2009, doi: 10.1111/j.1551-6709.2009.01023.x.

[9] N. H. R. Azamin, M. N. Taib, A. H. Jahidin, D. S. Awang, and M. S. A. M. Ali, "IQ level prediction and cross-relational analysis with perceptual ability using EEG-based SVM classification model," IAES International Journal of Artificial Intelligence, vol. 8, no. 4, pp. 436-442, Dec. 2019, doi: 10.11591/ijai.v8.i4.pp436-442.

[10] K. K. Brock, S. Mutic, T. R. McNutt, H. Li, and M. L. Kessler, "Use of image registration and fusion algorithms and techniques in radiotherapy: report of the AAPM Radiation Therapy Committee Task Group no. 132," Medical physics, vol. 44, no. 7, pp. e43-e76, 2017, doi: 10.1002/mp.12256.

[11] S. Ibrahim, N. E. A. Khalid, and M. Manaf, "CAPSOCA: Hybrid technique for nosologic segmentation of primary brain tumors," Indonesian Journal of Electrical Engineering and Computer Science, vol. 16, no. 1, pp. 267-274, Oct. 2019, doi: 10.11591/ijeecs.v16.i1.pp267-274.

[12] D. Mattes, D. R. Haynor, H. Vesselle, T. K. Lewellen, and W. Eubank, "PET-CT image registration in the chest using free-form deformations," in IEEE Transactions on Medical Imaging, vol. 22, no. 1, pp. 120-128, Jan. 2003, doi: 10.1109/TMI.2003.809072.

[13] J. P. W. Pluim, J. B. A. Maintz, and M. A. Viergever, "Mutual-information-based registration of medical images: a survey," in IEEE Transactions on Medical Imaging, vol. 22, no. 8, pp. 986-1004, Aug. 2003, doi: 10.1109/TMI.2003.815867.

[14] S. Abdelaziz and S. Lu, "K-means algorithm with level set for brain tumor segmentation," Indonesian Journal of Electrical Engineering and Computer Science, vol. 15, no. 2, pp. 991-1000, Aug. 2019, doi: 10.11591/ijeecs.v15.i2.pp991-1000.

[15] B. Huang, F. Yang, M. Yin, X. Mo, and C. Zhong, "A review of multimodal medical image fusion techniques," Computational and mathematical methods in medicine, 2020, doi: 10.1155/2020/8279342.

[16] S. Harish and G. F. A. Ahammed, "Integrated modelling approach for enhancing brain MRI with flexible pre-processing capability," International Journal of Electrical and Computer Engineering (IJECE), vol. 9, no. 4, pp. 2416-2424, Aug. 2019, doi: 10.11591/ijece.v9i4.pp2416-2424.

[17] A. C. Testa et al., "Imaging techniques for the evaluation of cervical cancer," Best practice \& research Clinical obstetrics \& gynaecology, vol. 28, no. 5, pp. 741-768, July 2014, doi: 10.1016/j.bpobgyn.2014.04.009.

[18] N. K. Kim, M. J. Kim, S. H. Yun, S. K. Sohn, and J. S. Min, "Comparative study of transrectal ultrasonography, pelvic computerized tomography, and magnetic resonance imaging in preoperative staging of rectal cancer," Diseases of the colon \& rectum, vol. 42, no. 6, pp. 770-775, 1999, doi: 10.1007/BF02236933.

[19] H. A. Lafta, Z. F. Hasan, and N. K. Ayoob, "Classification of medical datasets using back propagation neural network powered by genetic-based feature selector," International Journal of Electrical and Computer Engineering (IJECE), vol. 9, no. 2, pp. 13791384, Apr. 2019, doi: 10.11591/ijece.v9i2.pp.1379-1384.

[20] E. B. van de Kraats, G. P. Penney, D. Tomazevic, T. van Walsum, and W. J. Niessen, "Standardized evaluation methodology for 2-D3-D registration," in IEEE Transactions on Medical Imaging, vol. 24, no. 9, pp. 1177-1189, 2005, doi: 10.1109/TMI.2005.853240.

[21] A. Marathe, P. Jain, and V. Vyas, "Iterative improved learning algorithm for petrographic image classification accuracy enhancement," International Journal of Electrical and Computer Engineering (IJECE), vol. 9, no. 1, pp. 289-296, Feb. 2019, doi: 10.11591/ijece.v9i1.pp289-296.

[22] W. M. Wells III, P. Viola, H. Atsumi, S. Nakajima, and R. Kikinise, "Multi-modal volume registration by maximization of mutual information," Medical image analysis, vol. 1, no. 1, pp. 35-51, 1996, doi: 10.1016/S1361-8415(01)80004-9.

[23] A. M. Al-Smadi, M. K. Alsmadi, A. Baareh, I. Almarashdeh, H. Abouelmagd, and O. S. S. Ahmed, "Emergent situations for smart cities: A survey,', International Journal of Electrical and Computer Engineering (IJECE), vol. 9, no. 6, pp. 4777-4787, Dec. 2019, doi: 10.11591/ijece.v9i6.pp4777-4787.

[24] S. Binitha, and S. S. Sathya, "A survey of bio inspired optimization algorithms," International journal of soft computing and engineering, vol. 2, no. 2, pp. 137-151, 2012.

[25] M. K. Alsmadi, M. Tayfour, R. A. Alkhasawneh, U. Badawi, I. Almarashdeh, and F. Haddad, "Robust feature extraction methods for general fish classification,' International Journal of Electrical and Computer Engineering (IJECE), vol. 9, no. 6, pp. 51925204, Dec. 2019, doi: 10.11591/ijece.v9i6.pp5192-5204.

[26] M. Woźniak and D. Połap, “Adaptive neuro-heuristic hybrid model for fruit peel defects detection," Neural Networks, vol. 98, pp. 16-33, 2018, doi: 10.1016/j.neunet.2017.10.009. 
[27] M. Takruri, M. K. A. Mahmoud, and A. A.-Jumaily, "PSO-SVM hybrid system for melanoma detection from histo-pathological images," International Journal of Electrical and Computer Engineering (IJECE), vol. 9, no. 4, pp. 2941-2949, Aug. 2019, doi: 10.11591/ijece.v9i4.pp2941-2949.

[28] A. K. Anaraki, M. Ayati, and F. Kazemi, "Magnetic resonance imagingbased brain tumor grades classification and grading via convolutional neural networks and genetic algorithms," Biocybernetics Biomed. Eng., vol. 39, no. 1, pp. 63-74, 2019, doi: 10.1016/j.bbe.2018.10.004.

[29] H. Shahamat and M. S. Abadeh, "Brain MRI analysis using a deep learning based evolutionary approach," Neural Networks, vol. 126, pp. 218-234, 2020, doi: 10.1016/j.neunet.2020.03.017.

[30] M. Sajjad, S. Khan, K. Muhammad, W. Wu, A. Ullah, and S. W. Baik, "Multi-grade brain tumor classification using deep CNN with extensive data augmentation," Journal of computational science, vol. 30, pp. 174-182, 2019, doi: 10.1016/j.jocs.2018.12.003.

[31] P. Chang et al., "Deep-learning convolutional neural networks accurately classify genetic mutations in gliomas," American Journal of Neuroradiology, vol. 39, no. 7, pp. 1201-1207, 2018, doi: 10.3174/ajnr.A5667.

[32] W. Rahman, E. Hossain, R. Islam, H.-Ar-Rashid, N.-A-Alam, and M. Hasan, "Real-time and low-cost IoT based farming using raspberry Pi," Indonesian Journal of Electrical Engineering and Computer Science, vol. 17, no. 1, pp. 197-204, Jan. 2020, doi: 10.11591/ijeecs.v17.i1.pp197-204.

[33] Q. M. Shallal, Z. A. Hussien, and A. A. Abbood, "Method to implement K-NN machine learningto classify data privacy in IoT environment," Indonesian Journal of Electrical Engineering and Computer Science, vol. 20, no. 2, pp. 985-990, Nov. 2020, doi: 10.11591/ijeecs.v20.i2.pp985-990.

[34] N. V. Tanh, N. Q. Tri, and M. M. Trung, "The solution to improve information security for IoT networks by combining lightweight encryption protocols," Indonesian Journal of Electrical Engineering and Computer Science, vol. 23, no. 3, pp. 1727-1735, Sept. 2021, doi: 10.11591/ijeecs.v23.i3.pp1727-1735.

[35] K. Turzhanova, S. Konshin, V. Tikhvinskiy, and A. Solochshenko, "Performance evaluation of NB-IoT in-band deployment mode in suburban area," Indonesian Journal of Electrical Engineering and Computer Science, vol. 23, no. 3, pp. 855-862, Aug. 2021, doi: 10.11591/ijeecs.v23.i2.pp855-862.

[36] A. Rajbongshi, T. Khan, Md. M. Rahman, A. Pramanik, S. Md T. Siddiquee, and N. R. Chakraborty, "Recognition of mango leaf disease using convolutional neural network models: a transfer learning approach," Indonesian Journal of Electrical Engineering and Computer Science, vol. 23, no. 3, pp. 1681-1688, Sept. 2021, doi: 10.11591/ijeecs.v23.i3.pp1681-1688.

[37] E. Erwin, S. Saparudin, and W. Saputri, "Hybrid multilevel thresholding and improved harmony search algorithm for segmentation," International Journal of Electrical and Computer Engineering (IJECE), vol. 8, no. 6, pp. 4593-4602, Dec. 2018, doi: 10.11591/ijece.v8i6.pp4593-4602.

[38] Y. A. Fadil, B. Al-Bander, and H. Y. Radhi, "Enhancement of medical images using fuzzy logic," Indonesian Journal of Electrical Engineering and Computer Science, vol. 23, no. 3, pp. 1478-1484, Sept. 2021, doi: 10.11591/ijeecs.v23.i3.pp1478-1484.

[39] E. I. Zacharaki et al., "Classification of brain tumor type and grade using MRI texture and shape in a machine learning scheme," Magn. Reson. Med., vol. 62, no. 6, pp. 1609-1618, Oct. 2009, doi: 10.1002/mrm.22147.

[40] J. Cheng et al., "Enhanced performance of brain tumor classification via tumor region augmentation and partition," PloS ONE, vol. 10, no. 10, p. e0140381, Oct. 2015, doi: 10.1371/journal.pone.0140381.

[41] J. S. Paul, A. J. Plassard, B. A. Landman, and D. Fabbri, "Deep learning for brain tumor classification," Proc. SPIE, Med. Imag., Biomed. Appl. Mol., Struct., Funct. Imag., vol. 10137, p. 1013710, Mar. 2017, doi: 10.1117/12.2254195.

[42] P. Afshar, K. N. Plataniotis, and A. Mohammadi, "Capsule Networks for Brain Tumor Classification Based on MRI Images and Coarse Tumor Boundaries," ICASSP 2019-2019 IEEE International Conference on Acoustics, Speech and Signal Processing (ICASSP), 2019, pp. 1368-1372, doi: 10.1109/ICASSP.2019.8683759.

[43] M. G. Ertosun and D. L. Rubin, "Automated grading of gliomas using deep learning in digital pathology images: A modular approach with ensemble of convolutional neural networks," in Proc. AMIA Annu. Symp. Proc., vol. 2015, pp. 1899-1908, Nov. 2015.

[44] H. H. Sultan, N. M. Salem, and W. A.-Atabany, "Multi-Classification of Brain Tumor Images Using Deep Neural Network," in IEEE Access, vol. 7, pp. 69215-69225, 2019, doi: 10.1109/ACCESS.2019.2919122.

[45] S. K. Chandra and M. K. Bajpai, "Effective Algorithm For Benign Brain Tumor Detection Using Fractional Calculus," TENCON 2018-2018 IEEE Region 10 Conference, 2018, pp. 2408-2413, doi: 10.1109/TENCON.2018.8650163.

[46] Z. N. K. Swati et al, "Brain tumor classification for MR images using transfer learning and fine-tuning," Computerized Medical Imaging and Graphics, vol. 75, pp. 34-46, Jul. 2019, doi: 10.1016/j.compmedimag.2019.05.001.

\section{BIOGRAPHIES OF AUTHORS}

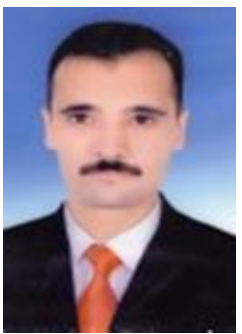

Ahmed Shihab Ahmed (D) $8 \mathrm{SC}$ P is a computer scientist specialized in the field of image processing and decision support systems. He received the four-year B.Sc. degree in Computer Science in 2000 from Al-Rafidain University College, Iraq. In 2015, he concluded a Master in Computer Science (MCS) from Middle East University, Jordan. He has been working as a programmer at University of Baghdad from 2004 until 2014 and then worked as an assistant lecturer at University of Baghdad from 2015 until now. His main research interests include: artificial neural network, image processing, decision support systems. He can be contacted at email: ahmedshihabinfo@ conursing.uobaghdad.edu.iq.

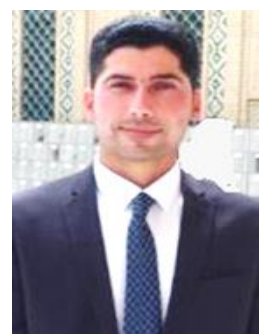

Hussein Ali Salah (DD 8I SC P received the four-year B.Sc. degree in Computer Science in 2000 from Al- Rafidain University College, Iraq. In 2004, he concluded a Master in Computer Science (MCS) from Baghdad University, college of science. He received the Ph.D. degree in Computer Science IT in 2016 from Politehnica' University of Bucharest, Bucharest, Romania. His main research interests include data mining, decision support system, web design and intelligent DSS. He has worked as a head of the computer systems department, Middle Technical University, Technical Institute-Suwaira, Wasit/Iraq from 2016 until now. He can be contacted at email: hussein_tech@mtu.edu.iq. 